\title{
Effectiveness of adaptive control of traffic light intersection on isolated multi-lane intersections
}

\author{
Roman Andronov ${ }^{*}, 1$, and Evgeny Leverents ${ }^{l}$ \\ ${ }^{1}$ Tyumen Industrial University, 625000 Tyumen, Russia
}

\begin{abstract}
The use of information technology in traffic management can increase the capacity of traffic intersections and reduce vehicle delays. In cities, adaptive traffic control at traffic lights intersections has been well established. Adaptive regulation is applied mainly on isolated traffic lights, where the traffic flow has a predominantly exponential distribution of intervals. It was established experimentally that the effectiveness of the method decreases with a supersaturated traffic flow, which makes it difficult to find a "cutoff point", a convenient moment for switching the traffic light signal. The article shows that at isolated multi-band intersections, the search for a convenient signal switching time is difficult at low loading levels. At uninsulated intersections, the incoming flow will have clear periods of time without vehicles, which makes the operation of adaptive traffic control in these conditions at a multi-lane crossing more efficient. This suggests the need for further study of the patterns of distribution of intervals between cars in urban environments at the approaches to non-isolated intersections and at the approach to intersections operating without coordination with other traffic lights.
\end{abstract}

\section{Introduction}

At present, the development of transport infrastructure is not the construction of new road junctions and not the expansion of roads, but the search for more advanced road traffic management options, including the introduction of information technologies and intelligent transport systems. This is the distribution of transport navigation systems on city streets, the introduction of automated traffic control systems, the synchronization of their data with the data of GPS navigators [1]. The organization of traffic mainly relies on the use of adaptive control of traffic lights, adapting to the changing parameters of traffic flows [2].

The transport infrastructure and methods of its organization should be harmoniously developed within the framework of the "Livable Cities" concept [3]. The development of information technology and intelligent systems can solve this problem.

Adaptive traffic control reduces vehicle delays at regulated intersections by $10-60 \%$ $[2,4,5]$. In many systems of adaptive regulation, the data collected by the detectors fixing the passage and the presence of vehicles are processed $[7,8,9]$. These systems include the Spektr 2.0, SCOOT and UTOPIA motion control systems.

\footnotetext{
* Corresponding author: aroma77777@mail.ru
} 
To substantiate the use of adaptive control, it is necessary to know the adequate law of the distribution of intervals between vehicles in order to determine the instant of switching of traffic lights. In most systems of adaptive control, this interval is assumed to be equal to or somewhat longer than 5 seconds [10].

The aim of the study is to conduct a numerical experiment using the «Monte Carlo» method. An signal-controlled intersection on which adaptive regulation is carried out with a different number of lanes on the approach to the stop line will be modeled.

\section{Matherials and methods}

In this study, on the basis of the model of the intersection work using the Monte Carlo method [2], a numerical experiment is conducted to investigate the efficiency of the signalcontrolled intersection with two and four lanes of transport to the stop line. As a result, the predicted average parameters were obtained: the cycle duration, the average delay of one car and the total percentage

of inclusion of the inhibit phase for the second direction with a duration of more than 120 s. 2 minutes are taken as a limit with respect to the comfortable waiting time for the enable signal.

Monte Carlo method has proved itself in simulating both complex systems and traffic flows at micro- and macro levels

The novelty of the study lies in the model of traffic flow through a signal-controlled intersection with adaptive control. Provided that a traffic situation is simulated multiple times with different initial conditions, this model makes it possible to calculate average values of queue parameters and vehicle delays.

\section{Experiment}

Recently, in the city of Tyumen, on the main arterial streets, the Spectrum 2.0 automated traffic control system is being introduced, which includes a unit for adaptive control of traffic light regulation. The primary information obtained from the TrafiCam video detectors is processed in the management center. The basic principle of the system is to find a "gap in the stream." The gap in the stream is the time interval of more than five seconds between cars. This is the optimal time for changing traffic-light signals and for the ultimate increase in throughput of the intersection.

In drawing up the model, an abstract intersection of one-way streets was taken. Switching the enabling signal and the beginning of the movement of vehicles occurred with the appearance of a "gap in the flow." The entire flow of vehicles on the intersected street is being passed through and when a "gap in the flow" appears, the traffic signal switches again.

Modeling was done without pedestrian traffic. General conditions satisfy the work of the system of adaptive control of the traffic light regulation "Spectrum 2.0".

A simulation of the approach of vehicles to the stop line for a short time interval was made on the assumption of an exponential distribution of intervals. It was assumed that the traffic flow is stationary for an hour. Passing cars through the stop line in traffic conditions with a stable traffic flow was based on the normal distribution law.

When the intersection is working at the capacity limit, the duration of the traffic light cycle with adaptive regulation can grow indefinitely and, some authors recommend limiting its limits [9]. But in this paper, based on the principle of the comparative simplicity of the model, the cycle time was not limited. 
The methodology presented in this study can be implemented in any programming language, in particular, Python.

\section{Result and discussion}

The total number of iterations of the model for each variant of the traffic situation was taken from the condition of the required reliability of $95 \%$ and the permissible error of the obtained average result no more than $10 \%$. The relevance of the data obtained is ensured by the general theory of traffic flow and on the basis of conducting preliminary experiments, in particular, presented in an article $[17,18]$.

The results of the numerical experiment are shown in Tables 1, 2 and Figures 1, 2. The final simulation data show the maximum, minimum and average duration of the traffic light cycle, the average delay time and the number of switching on the inhibitory phase lasting more than 120 seconds.

Table 1 The simulation results for the 2-way intersection

\begin{tabular}{|c|c|c|c|c|c|}
\hline $\begin{array}{l}\text { Intersection } \\
\text { load level }\end{array}$ & $\mathrm{T}_{\max }, \mathrm{s}$ & $\mathrm{T}_{\min }, \mathrm{s}$ & $\mathrm{T}_{\text {aver, }} \mathrm{s}$ & $\begin{array}{l}\% t_{\text {red }} \\
\text { more than } \\
120 \mathrm{~s}\end{array}$ & $\begin{array}{l}\text { Average } \\
\text { delay for } \\
1 \text { car, } \mathrm{s}\end{array}$ \\
\hline 0.52 & 95 & 30 & 59,8 & 0 & 16.8 \\
\hline 0.58 & 145 & 35 & 72 & 0 & 18.2 \\
\hline 0.65 & 180 & 40 & 79 & 0 & 20.6 \\
\hline 0.71 & 195 & 45 & 91,5 & 2 & 23.8 \\
\hline 0.77 & 285 & 55 & 124 & 12 & 28.6 \\
\hline 0.84 & 320 & 65 & 166 & 46 & 35.3 \\
\hline 0.90 & 725 & 90 & 242 & 70 & 52.6 \\
\hline
\end{tabular}

Table 2 The simulation results for the 4-way intersection

\begin{tabular}{|c|c|c|c|c|c|}
\hline $\begin{array}{c}\text { Intersection } \\
\text { load level }\end{array}$ & $\mathrm{T}_{\max }, \mathrm{s}$ & $\mathrm{T}_{\min , \mathrm{s}}$ & $\mathrm{T}_{\text {aver, } \mathrm{s}}$ & $\begin{array}{c}\% \text { tred } \\
\text { more than } \\
120 \mathrm{~s}\end{array}$ & $\begin{array}{c}\text { Average } \\
\text { delay for } \\
1 \text { car, } \mathrm{s}\end{array}$ \\
\hline 0.52 & 120 & 25 & 67 & 0 & 17.7 \\
\hline 0.58 & 140 & 30 & 66,5 & 0 & 19.8 \\
\hline 0.65 & 220 & 40 & 87,5 & 2 & 22.1 \\
\hline 0.71 & 245 & 50 & 103 & 6 & 25.4 \\
\hline 0.77 & 280 & 50 & 141 & 22 & 30.9 \\
\hline 0.84 & 425 & 65 & 189 & 50 & 41.3 \\
\hline 0.90 & 835 & 60 & 321 & 78 & 66.5 \\
\hline
\end{tabular}




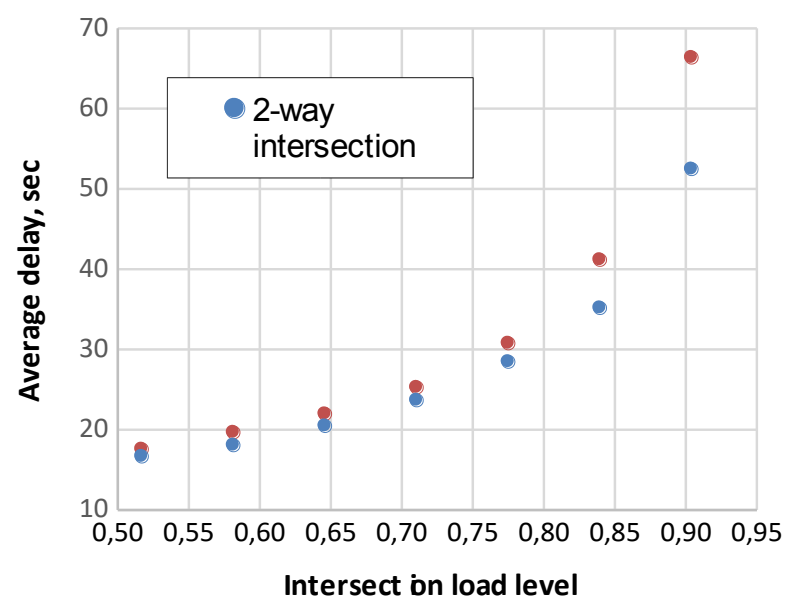

Fig. 1. Comparison of the average delay of one car at the intersection with adaptive regulation

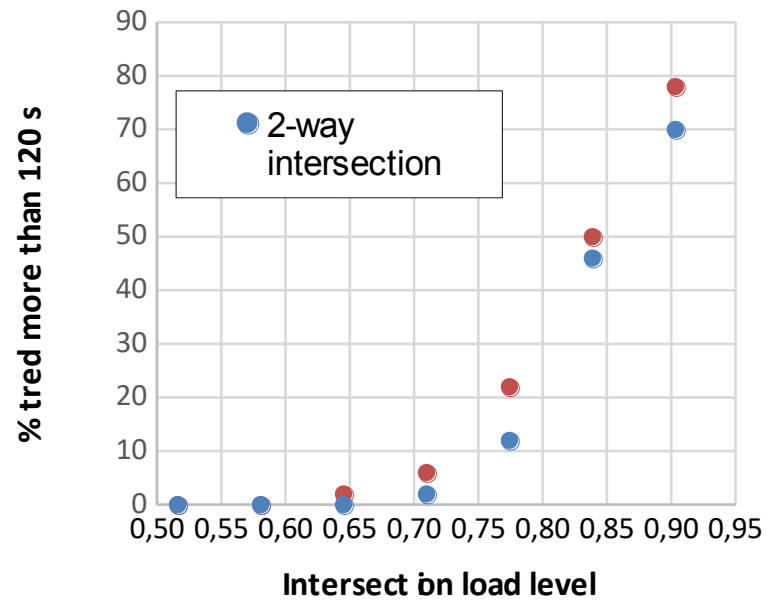

Fig. 2. Comparison of intersections by the criterion for the inclusion of a prohibiting phase lasting more than 120 seconds in the total regulation time

As can be seen from the above tables and graphs, with a multi-lane roadway, even at relatively small load levels, both the total duration of the traffic light cycle and the average delay time of one car are exceeded. Significantly increases the number of inclusions prohibitory signal lasting more than 120 seconds, which reduces the overall comfort when passing the intersection. In view of this, the switching of traffic lights will more often be carried out not at the point of "flow break", but at a fixed moment, as in the case of rigid regulation. Thus, the use of adaptive control on a multi-lane carriageway at isolated intersections significantly reduces its effectiveness.

In an urban environment with the relatively frequent location of adjustable intersections, isolated intersections are not predominant. In such a situation, the use of coordinated regulation is recommended. But under the condition of the relative difficulty of arranging such a regulation, the authors of the article make an assumption that adaptive control of traffic light regulation can also be used on non-isolated intersections. 
It is assumed that the regularities of the distribution of intervals will be different for isolated and uninsulated traffic light intersections because the inbound traffic flow of the latter is affected by the proximity of other intersections.

A preliminary study of incoming traffic to intersections in a single lane was conducted. The comparison was carried out to confirm or refute the correlation between the accuracy of the description of Poisson's law of the intervals between cars. The chi-square test was used. Intersections were considered uninsulated, on approaches to which the traffic light objects were located closer than $800 \mathrm{~m}$.

The data were checked for the presence between the coefficient of variation and other parameters for correlation according to the Student's t-test according to the method:

$$
t=\left|\frac{r_{x y} \sqrt{n-2}}{\sqrt{1-r_{x y^{2}}}}\right|
$$

where rxy is the correlation coefficient between the values; $n$ is the number of compared pairs $\mathrm{x}$ and $\mathrm{y}$;

The obtained value is compared with the critical value tcr for the significance level $\alpha=$ 0.05 and the number of degrees of freedom $f=n-2$ (For 36 pairs of values tcr $=2.03$ ). If the value of $\mathrm{t}>\mathrm{tcr}$ obtained using formula 1 , then the null hypothesis of the absence of any connection was rejected and the data should be checked for the existence of such a connection. The comparison results are shown in table 3.

Table 3 Comparison of approaches to intersections for the use of the exponential distribution for the Chi-square test of the city of Tyumen of the Russian Federation

\begin{tabular}{|c|c|c|c|c|c|}
\hline \multicolumn{3}{|c|}{ Isolated intersections } & \multicolumn{3}{|c|}{ nonisolated intersections } \\
\hline Street & $\begin{array}{l}\text { Chi- } \\
\text { square }\end{array}$ & $\%$ & Street & $\begin{array}{l}\text { Chi- } \\
\text { square }\end{array}$ & $\%$ \\
\hline st. Parkhomenko 1 & 7.8 & $5 \%$ & st. Herzen 1 & 3.9 & $27 \%$ \\
\hline st. Parkhomenko 2 & 3.5 & $47 \%$ & st. Herzen 2 & 2.8 & $42 \%$ \\
\hline st. Amurskaya 1 & 1.2 & $74 \%$ & st. Herzen 3 & 11.2 & $1 \%$ \\
\hline st. Amurskaya 2 & 0.9 & $92 \%$ & st. Herzen 4 & 9.1 & $3 \%$ \\
\hline st. Moscowsky trakt 1 & 4.3 & $37 \%$ & $\begin{array}{c}\text { st. Pervomaiskaya } \\
1\end{array}$ & 3.0 & $39 \%$ \\
\hline st. Moscowsky trakt 2 & 0.9 & $92 \%$ & $\begin{array}{c}\text { st. Pervomaiskaya } \\
2 \\
\end{array}$ & 1.2 & $74 \%$ \\
\hline st. Moscowsky trakt 3 & 1.2 & $76 \%$ & $\begin{array}{c}\text { st. Pervomaiskaya } \\
3 \\
\end{array}$ & 13.6 & $0 \%$ \\
\hline st. Moscowsky trakt 4 & 6.8 & $8 \%$ & $\begin{array}{c}\text { st. Pervomaiskaya } \\
4 \\
\end{array}$ & 3.0 & $40 \%$ \\
\hline st. Samartceva_1 & 1.3 & $72 \%$ & st. Permyakova 4 & 4.1 & $25 \%$ \\
\hline st. Samartceva_2 & 0.9 & $82 \%$ & st. Permyakova 5 & 3.9 & $28 \%$ \\
\hline st. Samartceva 3 & 0.7 & $87 \%$ & st. Melnikayte 2 & 6.7 & $8 \%$ \\
\hline st. Samartceva 4 & 6.9 & $7 \%$ & st. Melnikayte 3 & 6.7 & $8 \%$ \\
\hline st. Permyakova1 & 1.7 & $64 \%$ & st. Traktovaya 1 & 2.8 & $42 \%$ \\
\hline st. Permyakova2 & 3.1 & $38 \%$ & st. Traktovaya 2 & 8.6 & $3 \%$ \\
\hline st. Permyakova 3 & 8.3 & $4 \%$ & st. Traktovaya 3 & 0.1 & $99 \%$ \\
\hline st. Melnikayte 4 & 0.1 & $99 \%$ & st. Traktovaya 4 & 0.9 & $82 \%$ \\
\hline st. Melnikayte 5 & 2.0 & $57 \%$ & st. Traktovaya 5 & 25.2 & $0 \%$ \\
\hline st. Melnikayte 6 & 2.0 & $57 \%$ & st. Traktovaya 6 & 18.6 & $0 \%$ \\
\hline
\end{tabular}


The results of the study showed the presence of such a statically defined relationship, $(\mathrm{rxy}=3.8 ; \mathrm{t}=2.36$; $\mathrm{tcr}=2.03)$, but did not reveal its nature. We can definitely say that the exponential distribution describes worse the movement of such a flow and it is necessary to check the distribution of intervals between cars by other dependencies and statistical laws (Gauss, beta distribution, etc.)

Having the obtained model, it is possible to implement the modeling of traffic flow on real objects in various conditions.

The obtained results can be compared with other options for optimizing the movement or reconstruction of the intersection and find out which of them is more effective, especially in those conditions where construction and reconstruction works are difficult. (dense urban development)

\section{Conclusions}

Adaptive control of traffic lights is an effective means of increasing the crossing capacity and reducing vehicle delays. Its scope is limited to supersaturated flows at signalcontrolled intersection.

In the course of the study, a Monte Carlo numerical experiment was carried out on traffic modeling through 2 and 4 lanes intersection with adaptive control of traffic light regulation.

It was found that when operating adaptive regulation on a multi-lane carriageway of an isolated intersection (3-4 lanes in one direction), the efficiency of adaptive regulation decreases because there is little chance of a time interval of 5 seconds or more without cars on all lanes, even with a small intersection load. This will lead to a significant increase in the duration of the prohibiting phase, which will reduce the overall comfort when passing this intersection.

The article also showed a statistically significant difference in traffic conditions on approaches for isolated and non-isolated intersections.

It is assumed that with adaptive regulation at an uninsulated intersection, time intervals free from car passes will appear much more often, which will increase the overall efficiency of such an organization of movement at the intersection.

It is necessary to further justify the use of the desired type of distribution, for the correct modeling of a transport stream that approaches uninsulated intersections, which will later lead to an adjustment of the model of a transport intersection with adaptive regulation. This will allow obtaining more accurate results of the magnitude of vehicle delays and the queue length [2].

\section{References}

1. A. A. Testeshev, V. D. Timohovez, T. G. Mikeladze, "Identification of the Composition of Transport Streams for Remote Satellite Monitoring," IOP Conf. Ser. Mater. Sci. Eng., vol. 463, p. 022097, (2018)

2. R. Andronov, E. Leverents. Calculation of vehicle delay at signal-controlled intersections with adaptive traffic control algorithm. MATEC Web of Conferences, Proceedings of the IV International Young Researchers Conference "Youth, Science, Solutions: Ideas and Prospects (YSSIP-2017)", 25-27 (Tomsk, Russia, 2018).

3. V. R. Vuchic, Transportation for Livable Cities (Territorija budushhego, 2011) (in Russian) 
4. A.K. Debnath, Cities 37, 47-56 (2014)

5. V. Astarita, V.P. Giofrè, A.A. Vitale, ASRJETS 19, 1 (2016)

6. A.O. Evdokimov, A.V. Gorohov, D.Y. Lugov, I.R.J. 5, 55 (2015)

7. S. Djahel, Adaptive traffic management for secure and efficient emergency services in smart cities (PERCOM Workshops, IEEE, 2013)

8. E. A. Petrov, Transport strategy - XXI century, 12 (2011) (in Russian)

9. V. Erisov, V. Trushevski, EEJET 4, 9 (2014)

10. Ju.A. Kremenec, M.P. Pecherskij, M.B. Afanas'ev, Technical facilities for traffic organization (Akademkniga, 2005) (in Russian)

11. V. M. Polukarov, L. A. Jakushin, A Manual on the Design and Implementation of Automated Traffic Control Systems (MIA USSR, 1981) (in Russian)

12. ODM 218.6.010-2013. 5 YSSIP-2017 Methodical recommendations on the organization of road safety audit in the design and operation of highways MATEC Web of Conferences 143, 04008 (2018) (Informavtodor, 2014) (in Russian) https://doi.org/10.1051/matecconf/201814304008

13. N. Metropolis, S. Ulam, JASA 44, 247 (1949)

14. S. Sinha, Dynamics of urban traffic congestion: A kinetic Monte Carlo approach to simulating collective vehicular dynamics (COMSNETS, IEEE, 2016)

15. S. Tsiakmakis, TRR Journals 2572, 66-77 (2016)

16. V. V. Silyanov, Theory of traffic flows in the design of roads and traffic organization (Transport, Moscow, 1977)

17. ODM 218.6.003-2011. Methodical recommendations on the design of traffic lights on highways (Rosavtodor, 2013)

18. R. V. Andronov, Modeling of queues at regulated street intersections in a large city within the dense traffic] (Tyumen, 2007) 